INRA Prod. Anim., 2017, 30 (4), 351-362

\section{Quand l'élevage est garant de la conservation de milieux patrimoniaux}

\author{
S. LEMAUVIEL-LAVENANT ${ }^{1,2}$, R. SABATIER ${ }^{3}$
}

${ }^{\prime}$ UCN, UMR Ecophysiologie Végétale \& Agronomie, nutritions NCS, 14032, Caen, France 2 INRA, UMR EVA, 14032, Caen, France ${ }^{3}$ INRA, AgroParisTech, Université Paris-Saclay, UMR SAD-APT, 75000, Paris, France

Courriel : servane.lavenant@unicaen.fr

La conservation d'habitats semi-naturels est très souvent dépendante du maintien d'activités d'élevage dans des contextes pédoclimatiques contraignants. Cet article s'appuie sur deux exemples contrastés, les prairies de marais et les systèmes transhumants de Méditerranée, pour décrire l'importance de l'élevage dans le maintien des services environnementaux de milieux à haute valeur patrimoniale.

De nombreux écosystèmes à forte valeur patrimoniale ont une origine anthropique et résultent d'une activité agricole qui a maintenu le milieu ouvert (Huyghe et al 2014). La conservation de ces habitats ; prairies humides, pelouses, landes ou garrigues, est généralement conditionnée par le maintien d'une activité d'élevage extensive (Ostermann 1998, Halada et al 2011). Cette activité oriente les communautés végétales et faunistiques mais permet l'expression d'une biodiversité sauvage qui, associée aux biotopes, constitue des écosystèmes dits « semi-naturels ».

Les éleveurs sont les porteurs des services de production agricole, mais ils portent également d'autres services environnementaux et culturels dont bénéficie une population allant au-delà des seuls consommateurs de produits animaux. Les milieux semi-naturels abritent en effet de nombreuses espèces rares de la flore et de la faune. De par la grande biodiversité qu'ils accueillent et les paysages ouverts auxquels ils contribuent, les milieux ouverts offrent des services culturels notamment dans le cadre de nombreuses réserves et parcs naturels. Enfin, les prairies permanentes et les parcours (réseaux ouverts de pacage) sont à l'origine de services de régulation comme la séquestration du carbone (Petri et al 2010), la régulation des crues dans le cas des prairies humides (Maltby et Acreman 2011, Bremond et al 2013, Maltby et al 2013) ou la prévention des incendies dans le cas des parcours méditerranéens (Moreira et al 2001). Il s'agit alors de concilier services de support (habitats pour la biodiversité), de régulation et services socio-culturels avec les services de production agricole. Dans bien des cas, l'éleveur devient ainsi, consciemment ou non, un gestionnaire de milieux semi-naturels.

Les activités d'élevage en milieu seminaturel sont rendues plus difficiles par une moins bonne accessibilité aux parcelles, une moindre production et parfois une périodicité marquée de la production (hors inondation, hors sécheresse, hors neige...) (Maltby et Acreman 2011, Bremond et al 2013, Reine et al 2014). Ces difficultés ont entraîné deux tendances opposées : une partie des prairies a été « améliorée » au sens agronomique par un travail du sol, par fertilisation, drainage des zones humides ou irrigation des zones sèches, une autre partie a été abandonnée au profit de parcelles plus faciles à exploiter et souvent plus proches des bâtiments. Les milieux ouverts, d'origine agricole, sont aujourd'hui encore mis en danger par ce double mouvement d'intensification et de déprise agricole (Henle et al 2008).

La synthèse qui est présentée dans cet article est issue d'une expertise collective menée par l'INRA (Dumont et al 2017, ce numéro) et se place dans le cadre conceptuel de l'analyse des services rendus par l'élevage et ses produits, observés sous l'angle de cinq interfaces : $i$ ) les intrants, ii) le travail et l'emploi, iii) les marchés, $i v)$ les enjeux sociaux et culturels et $v$ ) l'environnement et le climat. De par l'aspect semi-naturel des écosystèmes concernés par cet article, l'aspect environnemental constitue l'interface la plus importante et qui sera donc la plus développée.

Les prairies humides et les systèmes transhumants de Méditerranée constituent deux exemples contrastés du point de vue des conditions édaphiques, climatiques et culturel de territoires à forts enjeux naturels où l'élevage joue un rôle central. Un excès d'eau dans le premier cas et les sècheresses annuelles dans l'autre conditionnent à la fois la végétation et les pratiques d'élevage. La clé d'entrée du cas d'étude marais est le territoire. Un territoire fortement anthropisé qui est le siège d'une diversité d'activités en interactions, parmi lesquelles l'élevage joue un rôle central dans le maintien des milieux ouverts. La deuxième situation correspond à un système ovin transhumant en zone méditerranéenne utilisant de manière opportuniste une diversité de ressources semi-naturelles en s'adaptant à leur temporalité. Dans cette seconde partie c'est le système d'élevage, par nature multi-territorial, qui constituera le fil conducteur.

Pour ces deux exemples, les activités d'élevage sont garantes de la conservation de milieux à haute valeur patrimoniale naturelle et culturelle mais sont aujourd'hui très fragiles. L'élevage en marais ou l'élevage craven se sont construits dans des contextes historiques, naturels et sociaux forts qui doivent être pris en compte pour assurer leur pérennité et donc les services qu'ils rendent.

\section{1 / Les enjeux de l'élevage en territoires de marais}

Bien que les territoires de marais soient aujourd'hui considérés comme des sanctuaires de biodiversité, notamment pour l'avifaune, ils n'en sont pas moins des milieux anthropisés résultant parfois 
d'aménagements de grande ampleur remontant jusqu'au moyen-âge. Les usages traditionnels des marais se sont vus modifiés au cours du $\mathrm{XX}^{\mathrm{e}}$ siècle en Europe, soit par drainage et conversion en cultures soit par abandon (Poschlod et al 2005). L'abandon des prairies humides conduit à la mise en place d'une succession secondaire entrainant le boisement progressif des marais (Opdekamp et al 2012, Menichino et al 2016).

Sous la seule appellation « prairies humides » les habitats sont aussi diversifiés que leur origine (inondation naturelle ou anthropique), leurs conditions pédoclimatiques et leur gestion agricole. La composition floristique de prairies humides ne se distingue parfois d'une prairie au sol relativement fertile et bien drainé, dite « mésophile », que par la présence de quelques espèces hygrophiles mais peut d'autres fois être très différente d'une prairie à vocation de production comme dans le cas des moliniaies, landes herbeuses dominées par la molinie bleue, jonchaies, prairies à forte proportion de joncs ou mégaphorbiaies, dominées par des dicotylédones. Afin de rendre compte de cette diversité de milieux rassemblés sous le terme de marais, l'analyse des marais s'appuiera sur trois territoires aux contextes pédoclimatiques et socio-culturels contrastés : le Marais Poitevin, les marais du Cotentin et les Culms au Royaume-Uni (encadré 1, figure 1).

\section{1 / Contexte}

L'histoire des marais est celle de territoires façonnés par l'Homme. Les marais, considérés comme des espaces insalubres, foyers du paludisme, et inutiles sont asséchés dès le moyen âge. Les premières opérations de drainage accompagnées d'aménagements hydrauliques sont conduites entre le $\mathrm{X}^{\mathrm{e}}$ et le $\mathrm{XIII}^{\mathrm{e}}$ siècle puis au cours des XVII ${ }^{\mathrm{e}}$ et XVIII ${ }^{\mathrm{e}}$ siècles pour les marais Poitevin, au cours des $\mathrm{XVIII}^{\mathrm{e}}$ et $\mathrm{XIX}^{\mathrm{e}}$ siècles pour les marais du Cotentin et depuis le XVIII ${ }^{e}$ siècle pour les culms du Devon. À partir des années 1970 les prairies sont massivement retournées notamment au profit des cultures de maïs fourrager (Duncan et al 1999). La transformation des marais et les risques environnementaux associés ont été à l'origine de l'implication ou de la création d'organismes en charge de la gestion ou de la protection de l'environnement. En France, ce rôle d'animation est porté par les Parcs Naturels Régionaux (PNR). Le PNR du Marais Poitevin est ainsi créé en 1979 et le PNR des marais du Cotentin et du Bessin en 1991. Ces PNRs ont un rôle de conciliation et de médiation entre les différents acteurs du territoire. Ils animent les démarches agroenvironnementales, et sont opérateurs de sites Natura 2000 et
Encadré 1. Présentation des trois sites utilisés pour illustrer les territoires de marais européens.

Le Marais Poitevin est la plus grande zone humide française de la façade Atlantique (près de 100000 ha). Il est issu de l'érosion d'un plateau calcaire exondé lors de la dernière grande glaciation puis recouvert d'alluvions et de sédiments constituant une argile grise, la bri. II se divise en trois grandes entités paysagères : le marais mouillé, espace bocager, le marais desséché mis en culture et la zone littorale.

Les marais du Cotentin et du Bessin forment un isthme reliant la presqu'île du Cotentin au continent. Ils sont issus de la transgression flandrienne qui a creusé de larges vallées comblées pour partie par des tangues et sables puis par de la tourbe (Boujot 2003). Les marais de certaines vallées sont aujourd'hui encore constitués de sols tourbeux alors que dans d'autres vallées, la tourbe a été recouverte d'argiles et de limons.

Les Culms sont des marais situés dans le nord-ouest du comté du Devon et le nord-est du comté de Cornouailles. Les "Culms " sont localisés sur un socle rocheux les « Culm measures » issu de la période carbonifère. Le soussol est principalement constitué de schistes et de grès et a pour originalité la présence occasionnelle de charbon anthraciteux. Les sols sont argileux, acides, peu drainants et par conséquent gorgés d'eau (Gardiner 2009). Trois rivières principales le Taw, le Torridge et le Tamar sont à l'origine des zones humides du «lowland».

Figure 1. Localisation des sites choisis pour décrire les territoires de marais (Marais du Cotentin et du Bessin, Marais Poitevin et Culms : polygones bleus) et les systèmes transhumants de Méditerranée (La Crau : polygone vert).

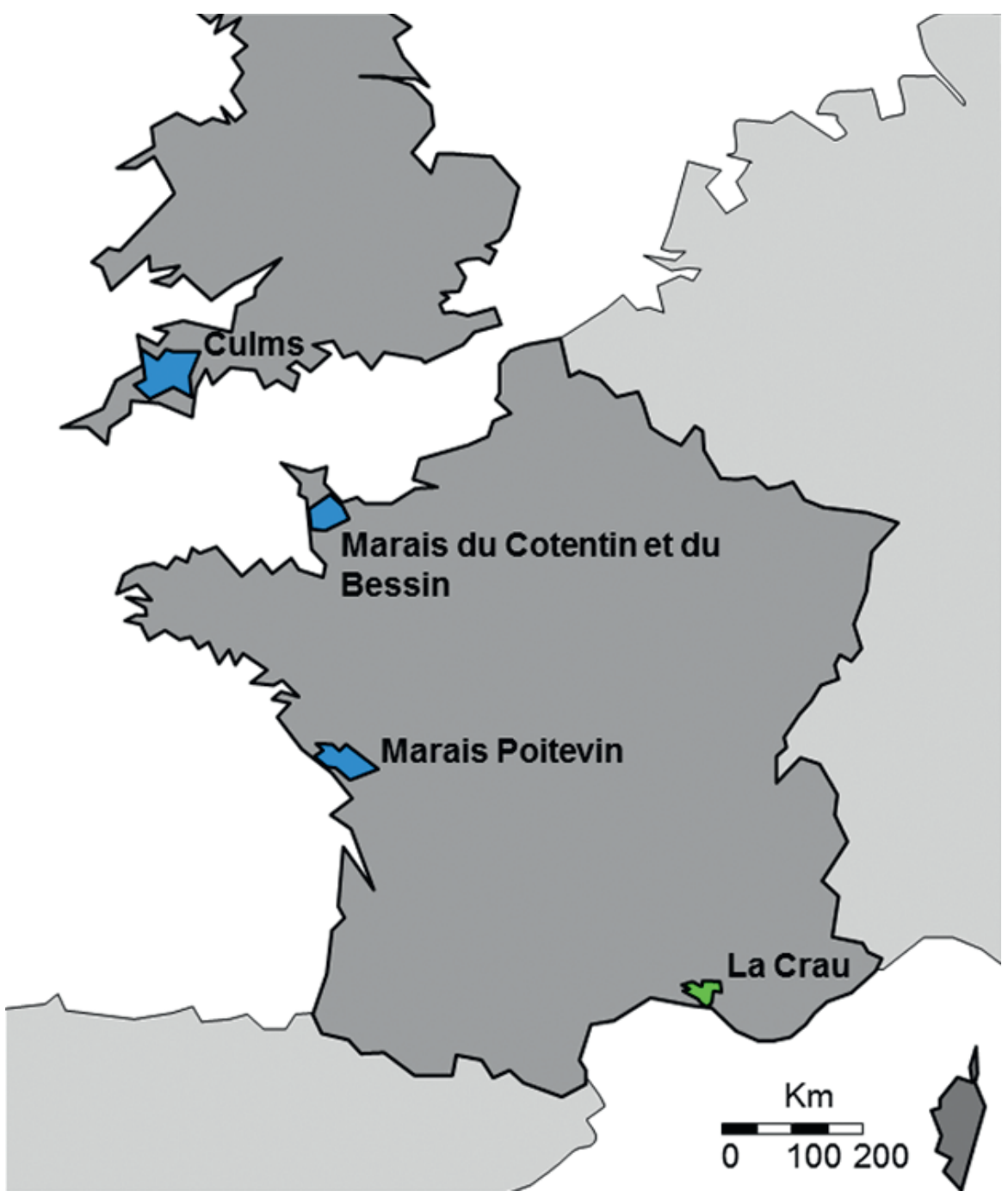


porteurs de Schémas d'Aménagement et de Gestion des Eaux (SAGE). Dans le Devon, le rôle de préservation des habitats est assuré par une association de protection de l'environnement, le « Devon Wildlife Trust », qui fait le lien entre les différents acteurs.

Les marais accueillaient autrefois un bétail diversifié au pâturage : bovins, chevaux, cochons, moutons, canards et oies, mais sont aujourd'hui principalement utilisés pour l'élevage bovin. Le Marais Poitevin est caractérisé par l'élevage bovin allaitant, avec pour races principales la charolaise, la limousine et la blonde d'aquitaine ainsi que deux races locales plus minoritaires : la nantaise et la maraîchine. Les marais du Cotentin sont spécialisés dans l'élevage bovin lait, avec essentiellement les races holstein et normande. Ce territoire connaît un essor de l'agriculture biologique. Les marais communaux dont certains sont encore en gestion collective occupent encore une portion importante dans les deux territoires de marais français. Les prairies des Culms sont traditionnellement entretenues grâce à l'élevage très extensif d'une race bovine à viande, la « ruby red cattle » (Gardiner 2009).

Les marais sont caractérisés par une gestion collective de l'eau, en termes de hauteurs et de durée de la période d'inondation (photo 1), qui nécessite une conciliation entre de multiples acteurs aux intérêts parfois conflictuels. Ainsi, les chasseurs au gabion, les pêcheurs et les plaisanciers souhaitent une durée d'immersion importante. À l'inverse, les céréaliers ne peuvent se permettre de voir leurs cultures inondées. Les éleveurs sont eux dans une situation intermédiaire : devant attendre le ressuyage des marais au printemps et une portance suffisante pour amener leurs animaux au pâturage ou pour faucher mais pouvant également supporter des périodes courtes d'inondation (voire en bénéficiant lorsque celles-ci permettent de compenser des sécheresses estivales). La gestion de l'eau est réalisée par des syndicats de marais par la manœuvre des ouvrages hydrauliques. Là aussi PNRs et associations jouent un rôle clef dans la médiation entre différents usagers.

Les inondations des prairies, autrefois perçues comme bénéfiques par les éleveurs car elles fertilisaient les terres, sont aujourd'hui moins bien vécues car elles rendent l'exploitation beaucoup plus contraignante. La production de fourrage en marais est conditionnée par les périodes de submersions-immersions et si cette production de fourrage est plus tardive qu'en plaine, sa croissance se maintient au cours de l'été. La production de fourrage des prairies hygrophiles des Marais Poitevin et des marais tourbeux du Cotentin atteignent des valeurs de 6 à $8 \mathrm{tMS} /$ ha ce qui les rend plus productives que les prairies mésophiles de ces territoires (Bonis 2004). La diversité floristique et la proportion en dicotylédones des prairies inondables permettent également de maintenir une bonne qualité fourragère au cours de la saison (Bonis 2004, Fillol et al 2015, Diquelou et al 2016). Les prairies pâturées peuvent néanmoins accueillir d'importantes proportions de joncacées, parfois valorisées sous forme de paillage, mais qui représentent un des freins à l'exploitation des marais pour l'élevage.

Le maintien de l'élevage dans ces trois zones de marais est aujourd'hui une préoccupation majeure des PNRs français comme du «Devon Wildlife Trust» en Angleterre. Le Marais Poitevin a conservé des pratiques très traditionnelles comme des déplacements en barque du bétail, il reste néanmoins sous la menace du retournement des prairies pour les cultures céréalières, le maintien des prairies humides n'étant dû qu'aux aides agroenvironnementales et au développement touristique. L'élevage est bien ancré dans les marais du Cotentin et du Bessin et les mesures agroenvironnementales permettent de maintenir des pratiques extensives sur les prairies inondables. La situation est beaucoup plus préoccupante dans les Culms où les surfaces des « Culm grasslands » ont régressé de manière drastique soit à cause de leur abandon et enfrichement, soit, majoritairement, du fait d'une intensification de l'agriculture (Gardiner 2009). Le « Devon Wildlife Trust » accompagne les agriculteurs par des aides financières et techniques pour enrayer la disparition de ces prairies humides mais aussi amorcer leur restauration.

\section{2 / Services environnementaux}

Parallèlement à leur utilisation pour l'élevage, les marais rendent des services environnementaux comme l'habitat pour la biodiversité ou la régulation des crues et de la qualité de l'eau.

Les marais accueillent ainsi des végétations diversifiées en fonction des contextes édaphiques (sols tourbeux versus minéraux) et hydrologiques et de la gestion (fauche vs pâturage, intensité de gestion) : prairies, roselières, mégaphorbiaies, caricaies, prairies tourbeuses, bas marais alcalins, végétations aquatiques et végétations halophiles. Parmi ces végétations, de nombreux habitats sont identifiés comme étant d'intérêt patrimonial au titre de la directive « Habitat » (Directive 92/43 CEE Annexe 1). Le Marais Poitevin a pour originalité une flore de végétations saumâtres avec l'emblématique jonc de Gérard dans les zones intermédiaires entre marais mouillés et marais desséchés (Bouzillé 1992, Bouzillé et al 2001). Les Culms du sud-est de l'Angleterre sont caractérisés par un habitat relictuel, les « Culm grasslands » dont il ne reste plus aujourd'hui que 5000 ha. Dans ces trois marais, l'élevage, via la gestion extensive par le pâturage ou la fauche permet de maintenir la végétation à un stade herbacé. La suppression des prairies par retournement, risque majeur dans le Marais Poitevin, mais aussi leur intensification par hausse de la fertilisation, conduit à la banalisation de la flore et par conséquent de la faune associée.

Photo 1. Le "marais blanc ", les marais du Cotentin et du Bessin sont inondés pendant l'hiver (@ S. Lemauviel-Lavenant).

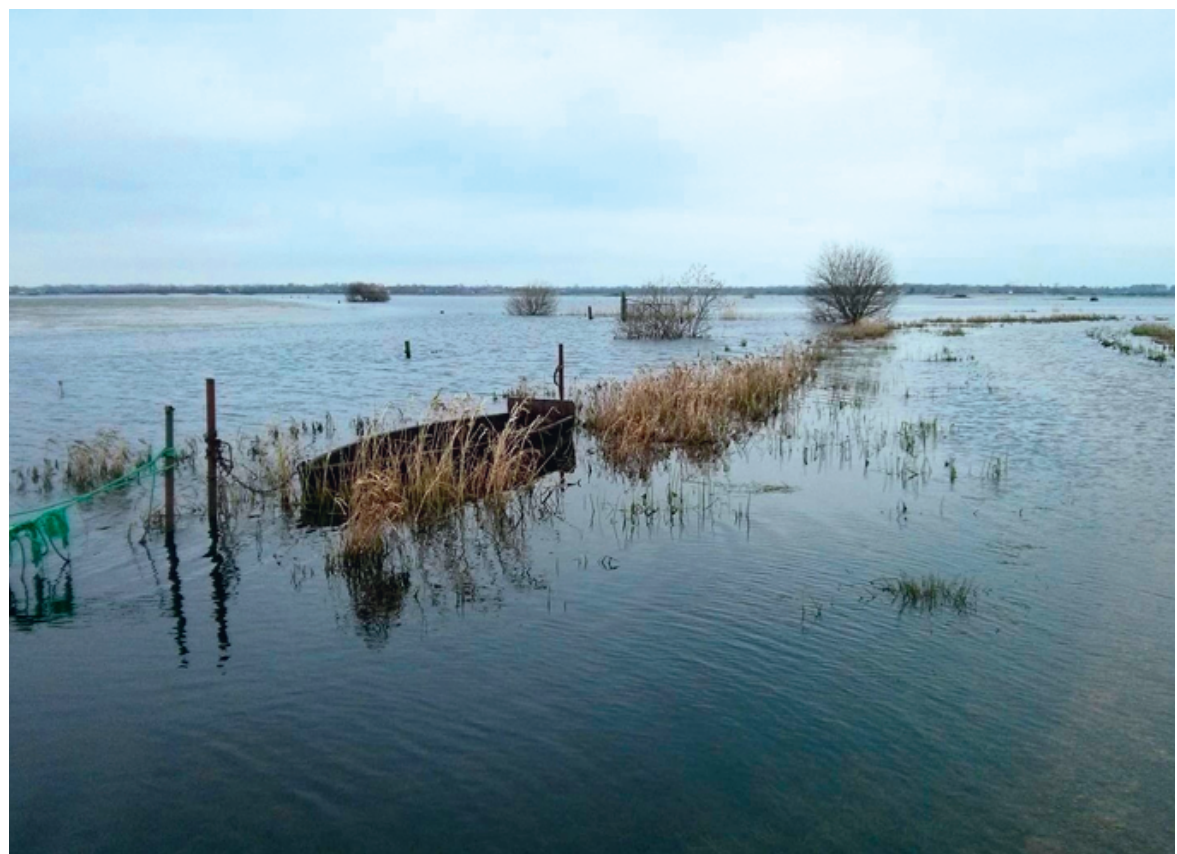


L'abandon des parcelles de prairies qui est une menace pour les trois sites et particulièrement pour les Culms conduit à la fermeture du milieu par des espèces ligneuses et donc à la disparition des habitats patrimoniaux et de la faune qu'ils accueillent. De manière plus indirecte, les éleveurs sont des acteurs clés de la gestion de l'eau et leur retrait entraînerait une modification des conduites de gestion de l'eau, comme l'entretien des fossés, qui ont abouti aux paysages actuels et à leur valeur patrimoniale.

La présence importante des échassiers constitue probablement la caractéristique principale qui a permis de focaliser sur la protection de ces zones humides. Quelques espèces emblématiques comme le courlis cendré, le vanneau huppé, le chevalier gambette, la barge à queue noire ou la bécassine des marais, nichent dans les prairies de marais et dépendent donc d'une activité d'élevage (Durant et al 2008). La gestion agricole des prairies est nécessaire pour les limicoles afin de conserver le milieu ouvert mais doit généralement être extensive afin de limiter les impacts directs tels que le piétinement des nids (Sabatier et al 2010). La « fauche tardive (après le 25 juin) sans fertilisation » et la « fauche très tardive (après le 25 juillet) sans fertilisation » sont ainsi des mesures agroenvironnementales largement adoptées en marais du Cotentin et du Bessin. Les marais accueillent aussi des espèces paludicoles nicheuses patrimoniales comme le butor étoilé, la cigogne blanche, le busard des roseaux, le busard cendré et la marouette ponctuée (Debout et al 2003). Outre les espèces nicheuses, les marais constituent des étapes pour les oiseaux d'eau migrateurs.

Les marais représentent aussi des habitats intéressants pour l'entomofaune et les batraciens (Fiche ZNIEFF Marais du Cotentin et du Bessin, id 25000814812). L'entomofaune des marais est représentée par de nombreuses libellules (agrion gracieux, aeschne printanière, leste dryade, agrion de Mercure...), orthoptères (criquet des clairières, criquet ensanglanté, criquet palustre, conocéphale des roseaux...), coléoptères et papillons (noctuelle pudorine, ancre, damier de la succise, ou encore fritillaire des marais espèce emblématique des « culm grasslands »). Les marais sont aussi peuplés d'une riche faune batrachologique comprenant des espèces peu communes tels le crapaud calamite et le triton crêté.

Les prairies inondées peuvent constituer des frayères pour le brochet et l'anguille (classée en danger critique d'extinction selon UICN). Par ailleurs, les rivières, sont fréquentées par des poissons migrateurs (lamproies fluviatile et marine, grande alose, saumon atlantique...) remontant ces cours d'eau pour frayer. Les marais du Cotentin et du Bessin recèlent enfin un annélide peu commun : la sangsue médicinale.

La biodiversité des marais est largement reconnue et les territoires des marais intègrent des zones Ramsar issues de la convention internationale de protection des zones humides de 1971 (e.g. les 32500 ha de la Baie des Veys), des réserves Naturelles (e.g. Réserve Naturelle Nationale - RNN - de la Baie de l'Aiguillon, RNN de la Sangsurière et de l'Adriennerie, RNN de la Baie des Veys, ou la réserve nationale du «Dunsdon Farm » en Angleterre), des zones Natura 2000 ZPS (Zones de protections spéciales) et ZSC (Zones spéciales de conservation). Le marais mouillé des Marais Poitevin constitue également un site classé au titre des Grands Sites de France depuis 2010.

Les prairies de marais ont également un rôle important dans la qualité des eaux qui se dirigent vers les cours d'eau et les nappes et permettent de moduler les crues et les sécheresses. La qualité des eaux est un des enjeux majeurs des marais. Le suivi de la qualité de l'eau dans les marais de l'ouest a mis en évidence une nette diminution de la teneur en nitrate entre l'amont et l'aval des marais (Bonis et al 2008). Le fonctionnement des zones humides a pour particularité une forte activité de dénitrification qui permet de réduire la teneur en nitrate des eaux circulantes (Maltby et al 2013). En revanche la dénitrification engendre une libération de $\mathrm{N}_{2} \mathrm{O}$, un important gaz à effet de serre, dans l'atmosphère.

L'élevage peut aussi être source de dégradation de la qualité de l'eau du fait de la divagation du bétail sur les berges des fossés et cours d'eau. La mise en défens et la pose d'abreuvoirs prélevant l'eau des cours d'eau comme de pompes à nez activées par les animaux peut pallier ces pollutions mais impliquent un important surcoût, l'intérêt historique des canaux étant précisément de jouer un rôle de clôtures et d'abreuvoirs naturels. La fertilisation des parcelles constitue une seconde origine de dégradation de la qualité de l'eau.

Les prairies humides des «lowlands》 du sud-ouest de l'Angleterre font l'objet depuis quelques années d'une plus grande attention des pouvoirs publics du fait de leur patrimonialité. Cette attention s'est accrue depuis les fortes inondations de l'hiver 2012-2013 qui ont paralysé une partie du territoire pendant plusieurs semaines (SQW 2013). La gestion des risques d'inondation passe par la conservation, voire la restauration des milieux inondables pour protéger les zones vulnérables comme les zones urbanisées. Les marais sont généralement décrits comme des éponges ; en période pluvieuse ils atténuent les phénomènes de crues et de ruissellement et, à l'inverse, alimentent les cours d'eau en période sèche. Les marais seront amenés à jouer un rôle de plus en plus grand dans l'atténuation des évènements climatiques extrêmes et de la montée du niveau de la mer liés aux changements climatiques globaux. Toutefois la capacité des marais à retarder et à atténuer les crues est dépendante de la capacité à stocker l'eau et donc à ne pas être saturés pendant des périodes clés (Bullock et Acreman 2003, Maltby et Acreman 2011).

L'anoxie dans les sols immergés est également un frein à la minéralisation de la matière organique. Les sols des prairies humides et en premier lieu des prairies tourbeuses constituent des stocks importants de carbone et contribuent ainsi à mitiger les émissions de $\mathrm{CO}_{2}$ (Maltby et Acreman 2011). Cette séquestration du carbone est toutefois à relativiser car l'activité des microorganismes telluriques peut être accélérée pendant la période de ressuyage.

\section{3 / Vitalité territoriale}

Dans les territoires de marais qui n'ont pas été intensifiés, l'activité agricole est souvent moins rentable et fragilisée. La densité de population est très faible et la population est nettement vieillissante. Dans les Culms, ce sont parfois des propriétaires privés, passionnés par ces milieux relictuels qui ont acquis les prairies pour les conserver.

Les services récréatifs offerts par les marais permettent de redynamiser les territoires. Ils sont essentiellement liés à des paysages traditionnels peu urbanisés et à la présence de l'eau permettant l'existence d'une avifaune remarquable, des activités liées à l'eau comme les promenades fluviales, la pêche et la chasse. Les marais sont également les lieux d'origine de nombreuses races locales participant à l'identité des territoires (vache maraîchine et baudet du Poitou dans le Marais Poitevin, vache normande dans les marais du Cotentin et « ruby red cattle » dans les marais des Culms).

Une offre de tourisme vert s'est développée sur ces territoires. Dans le Marais Poitevin la Venise verte représente un site touristique important. De même les 
pratiques traditionnelles comme l'arrivée des animaux dans les communaux du Marais Poitevin constituent des éléments attractifs pour le tourisme. Les marais font aussi l'objet d'une animation de sensibilisation à la nature et l'environnement par les PNR (dans le marais du Cotentin et du Bessin ou le PNR du Marais Poitevin), les CPIE (centre permanent d'initiatives pour l'environnement) et autres organismes comme les « Wild Life Trusts » des comtés d'Angleterre. Enfin, les marais constituent généralement un enjeu culturel fort dans l'imaginaire avec de nombreuses légendes propres aux territoires.

\section{4 / Synergie et compromis}

Les marais sont des territoires aux enjeux variés synthétisés par les cinq interfaces d'une « grange » conceptuelle issue de l'expertise collective menée par l'INRA (Dumont et al 2017) (figure 2 et tableau 1). Ils sont caractérisés par de faibles niveaux d'intrants, particulièrement dans le cas des marais du Cotentin et du Bessin, du fait de pratiques extensives de gestion. Ils portent des marchés modestes mais caractérisés par des produits de qualité, le lait dans les marais du Cotentin et du Bessin et la viande pour les Culms et le Marais Poitevin, parfois valorisés via leur ancrage dans ces territoires patrimoniaux. Les emplois agricoles sont quantitativement marginaux mais l'élevage, particulièrement dans les deux marais français, permet, via la conservation des marais, de maintenir un tissu rural par des emplois indirects : tourisme, pêche ou encore syndicats de marais. Dans les Culms, la faible surface de marais limite le poids de l'élevage dans l'économie et l'emploi local. Le tourisme lié à la beauté scénique du paysage est pour les trois territoires décrits, un des enjeux socio-culturels forts. Il se conjugue avec des activités de pêche et de chasse. L'interface environnement et climat porte des enjeux très forts. Les marais, illustrés ici par trois territoires aux contextes variés, offrent des services majeurs comme l'accueil de la biodiversité, et notamment de l'avifaune, la régulation des crues, la qualité de l'eau ou encore la séquestration du carbone dans le sol (figure 2 et tableau 1).

Ce sont des territoires complexes de par l'intervention de nombreux acteurs qui interagissent autour de la gestion des niveaux d'eau. Parmi eux, les éleveurs jouent un rôle clé en gérant les prairies. La conservation de milieux ouverts est conditionnée par le maintien des agriculteurs dans ces espaces plus contraignants et plus complexes à exploiter. Certains éleveurs sont attachés à "leurs marais ", ils y apprécient le cadre de vie, pour d'autres le lien est beaucoup plus ténu et la pérennité des prairies est beaucoup plus fragile. Le maintien de l'élevage sur les marais peut alors passer par une valorisation de cet élevage et une nouvelle conception de la production.

Biodiversité et gestion des prairies sont intimement liées (Sabatier et al 2010, Gaujour et al 2012). L'extensification qui passe par la limitation des intrants, des chargements dans le cas des prairies pâturées et la fauche tardive est recherchée pour favoriser la biodiversité. Ces pratiques sont encouragées par des mesures agroenvironnementales qui permettent de compenser un manque à gagner.

La valorisation du travail des éleveurs en tant qu'acteurs de l'environnement, de la beauté scénique du paysage mais

Tableau 1. Atouts et impacts associés aux systèmes d'élevage en prairies humides.

\begin{tabular}{|c|c|c|}
\hline Interfaces étudiées & Effets positifs & Effets négatifs \\
\hline Intrants & - Autonomie fourragère élevée & $\begin{array}{l}\text { - Effets délocalisés production d'aliments } \\
\text { concentrés } \\
\text { - Fourrages hors marais } \\
\text { - Fertilisation minérale des prairies } \\
\text { les plus intensives }\end{array}$ \\
\hline Travail et emploi & $\begin{array}{l}\text { - Emplois agricoles quantitativement } \\
\text { marginaux } \\
\text { - Maintien tissu rural par emplois indirects : } \\
\text { tourisme, pêche, syndicats de marais }\end{array}$ & \\
\hline Marchés & - Produits locaux de qualité (marques parc) & \\
\hline $\begin{array}{l}\text { Enjeux sociaux } \\
\text { et culturels }\end{array}$ & $\begin{array}{l}\text { - Qualité nutritionnelle des produits à } \\
\text { l'herbe } \\
\text { - Esthétique du paysage } \\
\text { - Tourisme, Chasse, Pêche, Identité } \\
\text { culturelle }\end{array}$ & $\begin{array}{l}\text { - Conflits d'usage sur la durée des périodes } \\
\text { d'inondation }\end{array}$ \\
\hline $\begin{array}{l}\text { Environnement } \\
\text { et climat }\end{array}$ & $\begin{array}{l}\text { - Biodiversité « commune » et patrimoniale } \\
\text { - Habitats Natura } 2000 \\
\text { - Séquestration du carbone } \\
\text { - Qualité des eaux } \\
\text { - Régulation des crues } \\
\text { - Diversité domestique (races bovines) } \\
\text { - Services intrants }\end{array}$ & $\begin{array}{l}\text { - Contraintes liées à la mise en eau } \\
\text { - GES : } \mathrm{CH}_{4}+\mathrm{N}_{2} \mathrm{O} \text { lié à la dénitrification }\end{array}$ \\
\hline
\end{tabular}


Figure 2. Bouquet de services des élevages en prairie humide selon la " grange » de l'ESCo (Duru et al 2017, ce numéro). Le pentagone central figure un territoire ou un système d'élevage. Ceux-ci intègrent certains éléments paysagers (haies) et sont décrits par leurs caractéristiques : espèces et densités animales (symbolisées par un animal petit ou grand), modes d'alimentation (animal vert = nourri au pâturage ou à base de fourrages). Le mode d'usage des terres est représenté par un parcellaire à deux nuances de vert pour symboliser la diversité des prairies permanentes, les cultures étant représentées en jaune.

Ce système interagit avec cinq interfaces : les marchés, le travail et l'emploi, les intrants, l'environnement et le climat, et les enjeux sociaux et culturels. Des pictogrammes, explicités dans le tableau ci-dessus, symbolisent les principaux éléments concernés par ces interfaces. La nature et l'ampleur des effets sont représentées par une flèche sortante plus ou moins large et dont la couleur indique que ces effets sont positifs (flèche verte), négatifs (rouge) ou mitigés (hachures) ; dans ce cas, l'effet dominant est celui qui borde la flèche. Sur l'interface travail et emploi, nous distinguons les emplois directs en élevage des emplois indirects (tourisme... ; flèche vers le personnage en blanc). Les flèches entrantes indiquent l'existence de pressions liées au marché, à l'utilisation d'intrants exogènes (qui symbolisent les effets délocalisés de l'élevage), ou bien (sur l'interface environnement et climat) que les systèmes d'élevage bénéficient de services intrants (flèche verte) ou subissent la pression de facteurs environnementaux (contraintes liées à la mise en eau des marais, prédation..., hachures rouges).

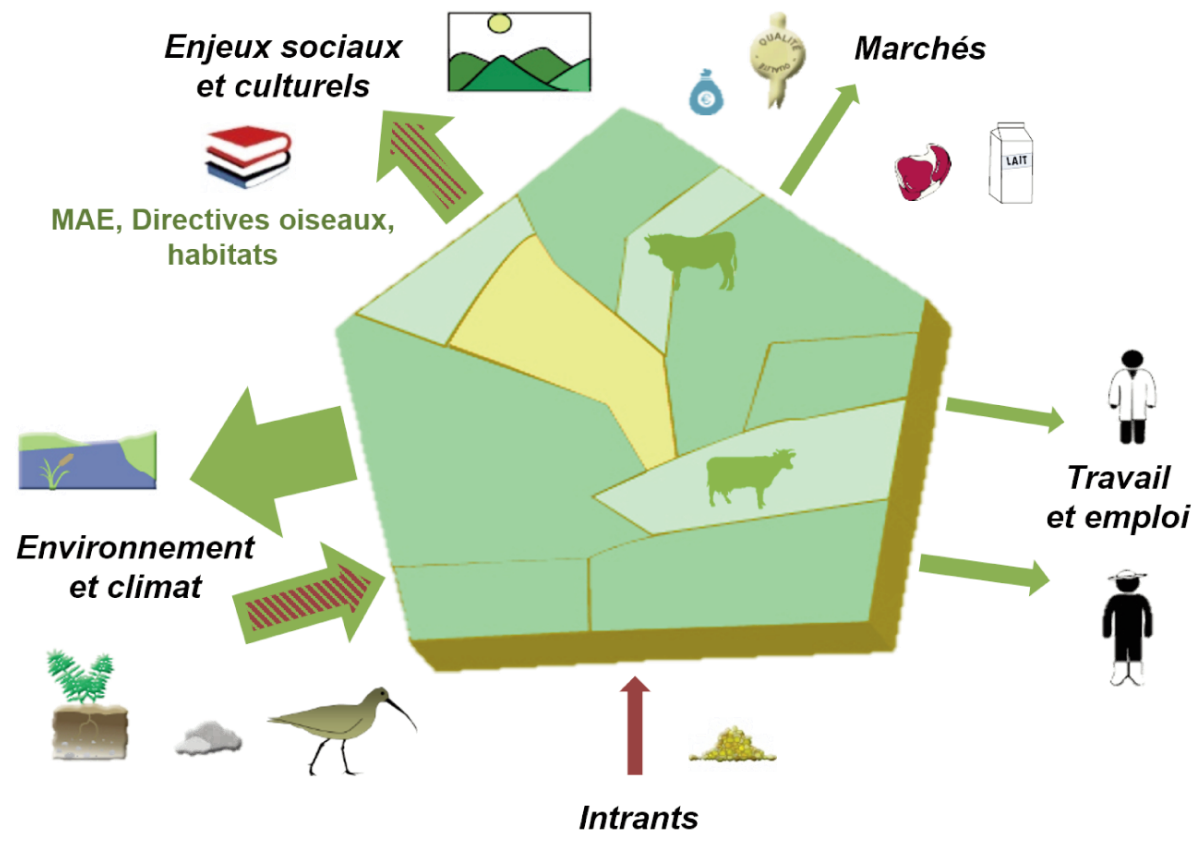

\section{Intérieur du territoire}

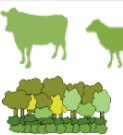

Animaux au pâturage

Haies, éléments paysagers

\section{Environnement et climat}

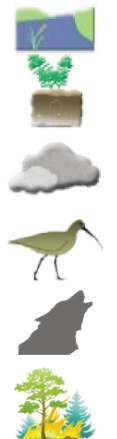

Eau de bonne qualité

Qualité des sols

Emission de GES et de particules fines dans l'air

Biodiversité

Présence du loup

Prévention des risques d'incendie

Travail et emploi

Emplois directs en élevage

if Emplois indirects (tourisme, IAA...)
Marchés

Produits animaux

Création de valeur monétaire

Foin de Crau

Produits sous signes de qualité

\section{Intrants}

Aliments concentrés

Enjeux sociaux et culturels

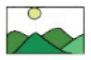

Esthétique paysagère

Aspects réglementaires et juridiques 
aussi en tant que producteurs de produits de qualité est une piste pour les maintenir sur ces terres difficiles à exploiter. La spécificité de l'élevage de marais peut être valorisée par la mise en place d'une production animale de qualité et de circuits courts comme la filière liée à la viande de race maraichine dans le Marais Poitevin ou celle liée à la viande de race « red ruby» dans le Devon mais une telle valorisation est loin d'être systématique.

Les manques à gagner issus des inondations sont souvent évalués à une échelle territoriale qui ne prend pas en compte l'hétérogénéité des impacts entre les exploitations (Bremond et al 2013). Une meilleure prise en compte des contraintes liées au caractère inondable des prairies est nécessaire pour que des mesures proposées aux éleveurs comme le paiement pour les services écosystémiques soient mieux acceptées (Bremond et al 2013).

\section{2 / Élevage craven, un exemple de système ovin transhumant en zone méditerranéenne}

À l'autre extrémité d'un gradient hydrique, la conservation de milieux semi-arides dépend elle aussi de la conservation d'une d'activité d'élevage. La plaine de Crau, située dans les Bouches-du-Rhône (figure 1) accueille un écosystème patrimonial, le Coussoul, considéré comme la dernière steppe Européenne. Il s'agit d'une végétation éparse se développant sur un sol oligotrophe peu profond et contraint par un climat méditerranéen chaud, sec et venteux (Buisson et Dutoit 2006) (photo 2). Pendant longtemps, cette zone sèche recouverte de galets siliceux a été considérée comme un milieu inutile et a été transformée. Outre l'occupation d'espaces pour l'industrie et les activités militaires, la plaine de Crau a vu son paysage transformé par les activités agricoles (Buisson et Dutoit 2006). Les cultures céréalières ou légumières comme la culture du melon, ainsi que le développement des vergers, notamment pour le pêcher, ont réduit l'espace des coussouls qui ne représentent aujourd'hui que de faibles surfaces. Ces systèmes transhumants portent les enjeux de protection des habitats patrimoniaux de la plaine de Crau et jouent également un rôle fondamental dans la préservation des milieux.

\section{1 / Contexte}

Les pelouses sèches de la plaine de la Crau composent un écosystème unique en France dont les caractéristiques sont intimement liées à l'activité d'élevage.

Photo 2. Le coussoul de la plaine de Crau (@ S. Masson).

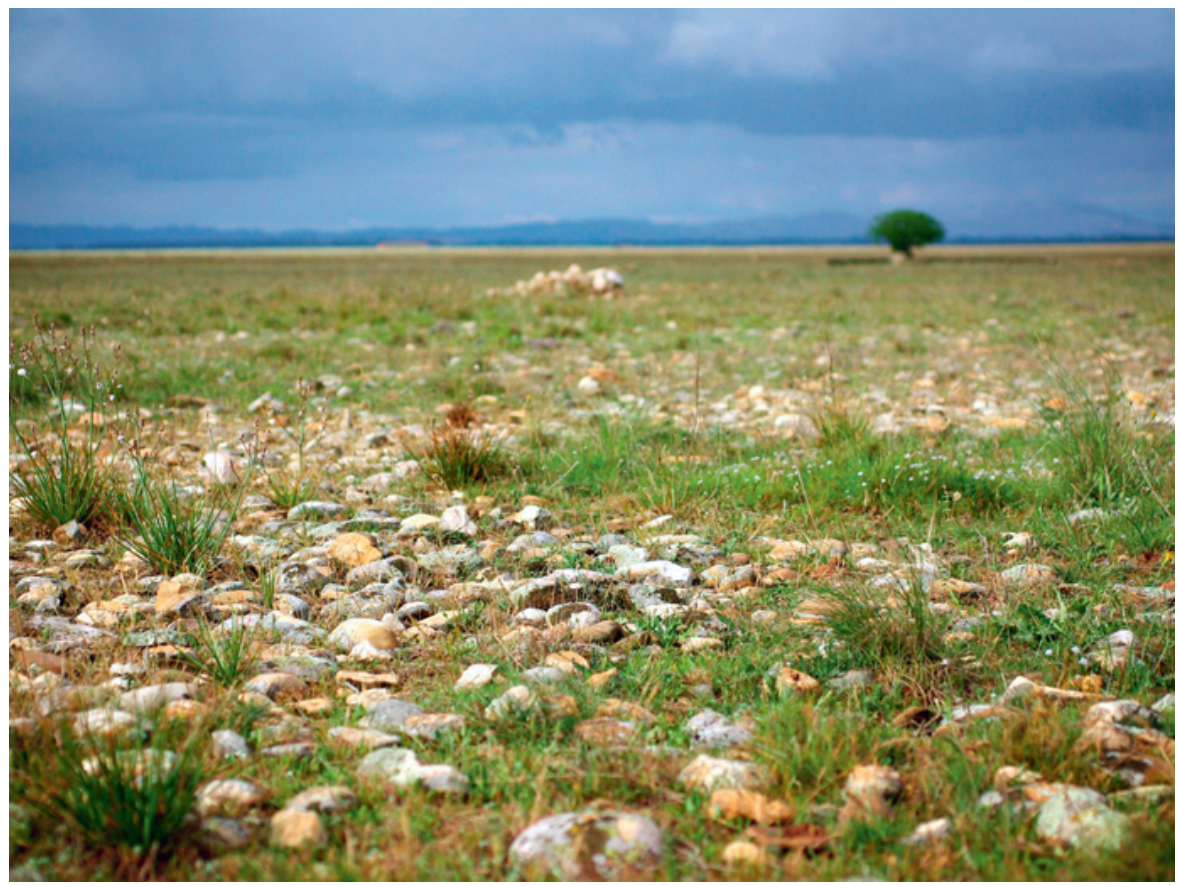

Ce milieu semi-steppique, le coussoul est à la base d'un système d'élevage ovin archétypal des élevages transhumants méditerranéens (Vincent 2011). Outre les coussouls, ce système mobilise 3 ressources complémentaires : les prés de fauche de la Crau irriguée, les collines méditerranéennes (notamment les Alpilles) et les prairies d'Alpage. Ce système se caractérise par un faible niveau d'intrant, une forte autonomie fourragère et une forte mobilité des troupeaux entre les différents milieux. Les terres sont louées à la saison, les éleveurs n'étant généralement propriétaires que de faibles surfaces de prairies de fauche.

La plaine de la Crau est certainement l'un des plus vieux territoires d'élevage ovin français avec des traces de bergeries datant de l'époque romaine (Badan et al 1995). Si les premiers élevages y étaient vraisemblablement sédentaires, ils deviennent transhumants à partir de la fin du Moyen Âge. L'utilisation de pâturages d'altitude en été permet en effet de contourner la sécheresse estivale. $\mathrm{Au} \mathrm{XVI}^{\mathrm{e}}$ siècle, de grands aménagements hydrauliques permettent d'irriguer une partie de la Crau avec l'eau de la Durance. Peu à peu, le coussoul y laisse place à une zone de prairies permanentes, connue aujourd'hui sous le nom de Crau irriguée, spécialisée dans la production de foin de qualité sous l'Appellation d'Origine Protégée « Foin de Crau ». En bordure de cette plaine, le massif des Alpilles, oscille entre activité forestière et agriculture. Dans cette zone d'élevage historique, le pâturage est longtemps considéré comme néfaste car responsable de dégâts à la forêt, de l'érosion et des inondations. Les éleveurs se voient finalement exclus des Alpilles à partir du XIX ${ }^{\mathrm{e}}$ siècle avec la promulgation du code forestier de 1827 puis la mise en place de la politique de restauration des terrains de montagne en 1860 (Tillier 2011). La situation perdure jusqu'à la fin des années 1980 où plusieurs incendies de grande envergure (montagne Sainte-Victoire, massif de la Sainte-Baume, Alpilles...) font prendre conscience des risques qu'entraînent l'embroussaillement des massifs provençaux. L'élevage ovin, est réintroduit dans les collines. Dans les Alpes également, la question environnementale est intimement liée au pâturage ovin. Ainsi, dans le massif du Queyras, comme en Provence, l'élevage a longuement été considéré comme néfaste à l'environnement. Au lendemain de la deuxième guerre mondiale, l'agriculture dans cette région enclavée aux conditions pédoclimatiques particulièrement hostiles peine à se moderniser. L'exode rural vide le territoire, entraînant l'abandon des formes traditionnelles d'agriculture. L'élevage ovin transhumant se développe alors sur ces terres agricoles délaissées pour devenir le principal mode de valorisation des terres en dehors des vallées.

Bien que composante essentielle de l'identité culturelle méditerranéenne, l'élevage ovin n'y a longtemps été vu qu'au travers de ses impacts négatifs sur l'environnement (sur-pâturage, érosion...). Le point de vue change à partir des années 1970-1980, les politiques publiques intégrant peu à peu l'élevage comme moyen de gestion, notamment dans le cadre de la lutte contre l'embroussaillement : afin de limiter les risques d'incendie en Provence, pour des questions paysagères dans les Alpes. 
Historiquement tourné vers la laine, ce système d'élevage est aujourd'hui orienté vers la production conjointe de viande et de foin de Crau. Le foin produit, sous l'AOP « Foin de Crau » est un foin réputé pour sa grande qualité. Principalement vendu pour l'alimentation en élevage équin, c'est une production à forte valeur ajoutée qui s'exporte non seulement en dehors des Bouchesdu-Rhône mais aussi en dehors de la France. Le système d'irrigation par gravité qui caractérise ce territoire permet des rendements élevés (10 à 11 t.ha $\left.^{-1} \cdot \mathrm{an}^{-1}\right)$ répartis en trois coupes.

La production ovine s'appuie sur une race rustique, la mérinos d'Arles, élevée pure ou en croisement avec des béliers de races à viande (île de France, berrichonne, charollaise) dont l'alimentation dépend de quatre ressources clés : prés, coussouls, colline et alpage. Le cycle annuel se décompose de la manière suivante, la mise bas d'automne se fait au retour d'alpage, sur les prés de fauche pâturés pour l'occasion dans le cadre d'une « quatrième coupe ». Ce pâturage $d$ 'hiver sur cette ressource de très bonne qualité permet d'assurer les besoins des brebis en lactation; il est également indispensable pour fertiliser les prés et assurer une bonne qualité du foin lors de la première coupe de printemps. Fin février, le troupeau quitte les prés de fauche pour les parcours de coussouls et les collines sur des ressources de moins bonne qualité. Enfin, les animaux passent l'été à l'alpage, sous la surveillance d'un berger après une transhumance opérée en camion au milieu du mois de juin.

\section{2 / Services environnementaux}

Dans les quatre espaces auquel il est associé, l'élevage ovin est au cœur des dynamiques agroécologiques et une de ses fonctions principales réside dans le maintien d'une diversité de services environnementaux.

En plaine de Crau, la patrimonialité des végétations de coussoul réside dans l'originalité de l'association d'espèces plus que dans la rareté des espèces végétales elles-mêmes. Parmi les végétations rencontrées, la « Pelouse méditerranéenne mésotherme de la Crau à Asphodelus fistulosus » (6220*) est identifiée d'intérêt communautaire et prioritaire au titre de la directive Natura 2000 Habitats, Faune, Flore.

En entretenant l'ouverture des milieux, le pâturage ovin est indispensable au maintien des habitats de nombreuses espèces patrimoniales, telles que le ganga cata, l'outarde canepetière ou le criquet rhodanien en plaine de Crau, l'aigle de bonelli dans les zones de collines ou le tétras lyre dans les Alpes. Un ajustement de l'intensité et des périodes de pâturage est néanmoins nécessaire pour favoriser les effets positifs de l'élevage par rapport aux effets négatifs comme par exemple le dérangement du tétras lyre pendant la phase de nidification (Patthey et al 2012).

La Crau et particulièrement les coussouls sont aujourd'hui reconnus pour leur patrimonialité et font l'objet d'une protection à travers des sites Natura 2000 aux titres des directives oiseaux et Faune Flore depuis les années 1990 et d'une réserve naturelle " $\mathrm{RN}$ des Coussouls de la Crau » créée en 2001.

La biodiversité sauvage peut en retour avoir des impacts négatifs sur l'activité d'élevage. L'arrivée de l'emblématique loup dans les Alpes et son impact sur les troupeaux a profondément modifié les métiers d'éleveur et de berger et entraîné de nombreuses modifications du système d'élevage dans son ensemble.

Le pâturage permet de lutter contre la fermeture des milieux, avec des conséquences paysagères (et donc touristiques) mais aussi un rôle de protection contre certains risques naturels comme les incendies dans les zones de collines en Provence ou la lutte contre les risques d'avalanche dans les Alpes.

La production de foin de Crau permet l'entretien d'un système d'irrigation par gravité vieux de près de 500 ans qui, audelà de son rôle agronomique, est également la source principale d'alimentation de la nappe phréatique locale. Les pratiques agricoles sur les prairies de fauche, basées sur de faibles niveaux d'intrants, ont peu d'externalités négatives sur la nappe, ce qui rend le système dans son ensemble favorable à la fourniture de ressources en eau de bonne qualité.

\section{3 / Vitalité territoriale}

Les Alpes et Alpilles sont en déprise agricole depuis la fin du $\mathrm{XX}^{\mathrm{e}}$ siècle et ont connu un exode rural important. $\mathrm{La}$ plaine de Crau est quant à elle soumise à une importante pression d'urbanisation et industrielle. Néanmoins le couplage de la production de foin de Crau et du pastoralisme ont jusqu'ici permis de préserver en partie le paysage et le patrimoine culturel. L'identité du territoire de la Crau est indissociable du métier de berger (Pelen 1985). Des fêtes de la transhumance ont vu le jour à Saint-Rémy de Provence et à Saint-Martin de Crau au milieu des années 1990 et témoignent de l'intérêt du grand public pour l'activité d'élevage. Ce regain d'intérêt se traduit également par une ouverture des profils des candidats au métier de berger à des personnes non issues du milieu agricole.

\section{4 / Synergies et compromis}

L'élevage craven s'appuie sur un système mêlant l'utilisation de plusieurs éco-agrosystèmes qu'il impacte différemment (figure 3 et tableau 2). L'utilisation d'intrants et d'énergie se concentre principalement sur les prairies de foin de Crau. Le transport des animaux lors de la transhumance induit également une dépense d'énergie. Des intrants, sous forme d'aliments concentrés sont également utilisés pour la finition des agneaux. Les emplois liés à l'élevage sont faible-

Figure 3. Bouquet de services en système d'élevage Craven selon la " grange » de l'ESCo (Duru et al 2017).

Le mode de représentation, l'interprétation des figurés et la signification des pictogrammes sont précisés en figure 2 .

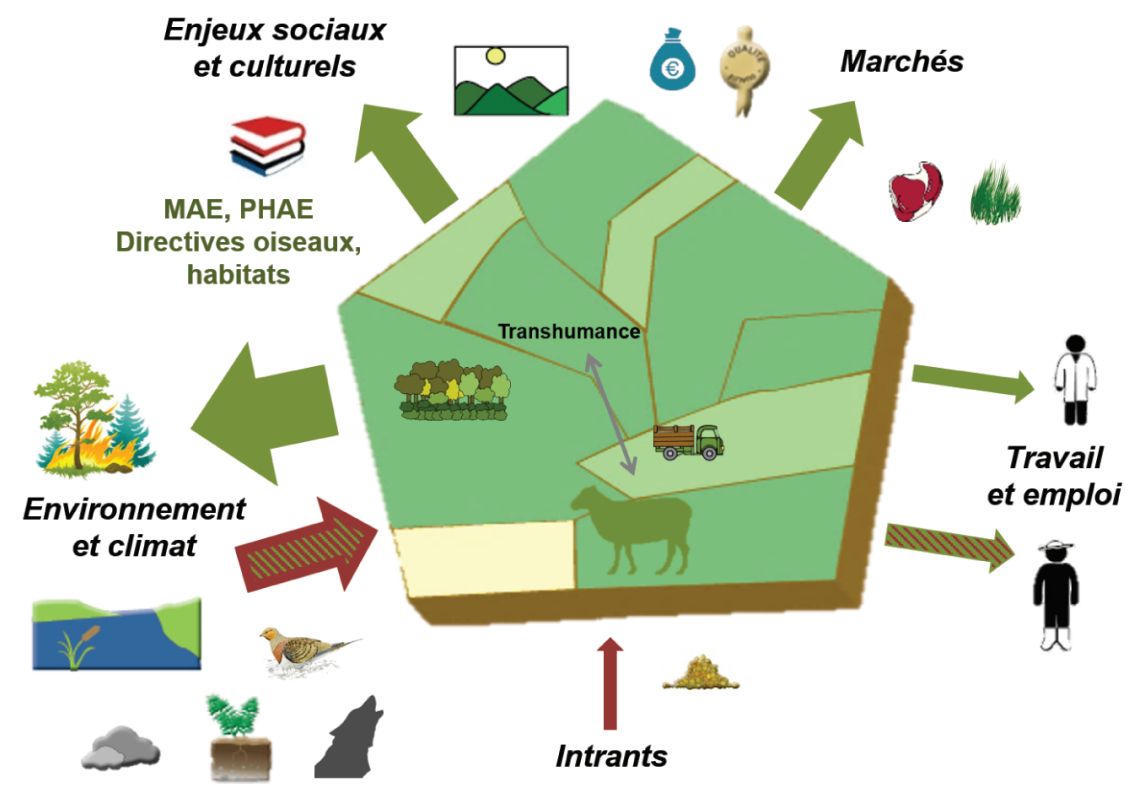


Tableau 2. Atouts et impacts associés au système d'élevage Craven.

\begin{tabular}{|c|c|c|}
\hline $\begin{array}{l}\text { Interfaces } \\
\text { étudiées }\end{array}$ & Effets positifs & Effets négatifs \\
\hline Intrants & - Autonomie fourragère élevée & $\begin{array}{l}\text { - Fertilisation minérale des prairies de foin } \\
\text { de Crau } \\
\text { - Énergie pour transport, fauche } \\
\text { - Aliments concentrés pour finition des } \\
\text { agneaux }\end{array}$ \\
\hline Travail et emploi & $\begin{array}{l}\text { - Emploi agricole quantitativement limité } \\
\text { mais satisfaction au travail } \\
\text { - Maintien tissu rural par emplois indirects : } \\
\text { tourisme, commercialisation produits }\end{array}$ & - Faible rémunération des emplois agricoles \\
\hline Marchés & $\begin{array}{l}\text { - Produits SIQO (label rouge) } \\
\text { - Foin de Crau AOP } \\
\text { - Grands troupeaux } \\
\text { - Valorisation en vente directe }\end{array}$ & $\begin{array}{l}\text { - Fragilité de la filière ovine, atténuée par } \\
\text { circuits locaux }\end{array}$ \\
\hline $\begin{array}{l}\text { Enjeux sociaux } \\
\text { et culturels }\end{array}$ & $\begin{array}{l}\text { - Qualité nutritionnelle produits à l'herbe } \\
\text { - Esthétique du paysage } \\
\text { - Tourisme } \\
\text { - Identité culturelle (bergers en Crau, } \\
\text { transhumance) }\end{array}$ & $\begin{array}{l}\text { - Morsures par chiens de protection } \\
\text { des troupeaux }\end{array}$ \\
\hline $\begin{array}{l}\text { Environnement } \\
\text { et climat }\end{array}$ & $\begin{array}{l}\text { - Biodiversité « commune » et patrimoniale } \\
\text { - Habitat remarquable (coussoul) } \\
\text { - Lutte contre fermeture des milieux et les } \\
\text { incendies } \\
\text { - Réalimentation de la nappe phréatique }\end{array}$ & $\begin{array}{l}\text { - Risques liés à la prédation, à l'érosion, } \\
\text { aux dérangements }\end{array}$ \\
\hline
\end{tabular}

ment rémunérateurs et assez marginaux mais apportent une qualité de vie qui s'est traduite par un regain d'intérêt ces dernières années pour le métier de berger. Ils permettent de maintenir un tissu rural. L'élevage craven, par le maintien du paysage et de pratiques patrimoniales induit des emplois dans le tourisme et le commerce des produits locaux. Le marché est caractérisé par une crise de la filière viande ovine d'une part mais par une bonne valorisation de certains produits comme le foin de Crau pour leur qualité et leur identité territoriale ou la viande via la vente directe. L'identité culturelle liée à ce système est forte et particulièrement liée aux pratiques d'élevage comme la transhumance, au métier de berger mais aussi aux paysages ouverts entretenus par les animaux. L'arrivée du loup s'est accompagnée de reconfigurations profondes du système et de différentes difficultés allant au-delà de la prédation en elle-même. Notamment l'utilisation de chiens pour protéger les troupeaux peut entraîner des conflits avec les promeneurs. Dans la plaine de la Crau, les éleveurs sont au cœur des enjeux d'environnement et de climat (figure 5 et tableau 2). La gestion des prairies irriguées permet quant à elle de réalimenter la nappe phréatique. Dans les collines et à l'alpage, l'entretien des parcours permet de lutter contre les incendies pendant l'été et contre les avalanches l'hiver. L'élevage peut induire un dérangement de certaines espèces et parfois induire une érosion des sols mais a globalement un effet environnemental très positif par la limitation de l'embroussaillement. L'importance de l'élevage dans la conservation de la biodiversité apparait notamment dans la gouvernance de la réserve naturelle des Coussouls de la Crau. Elle est en effet cogérée, classiquement par un conservatoire d'espaces naturels (CEN), le CEN Provence-AlpesCôte-d'Azur, et de manière plus remarquable, par la Chambre d'agriculture des Bouches-du-Rhône. La mesure de l'importance de l'activité d'élevage a également été prise au niveau des sites Natura 2000, puisqu'un projet LIFE nature " Crau sèche », initié en 1994, ciblait des aides aux éleveurs et leur a permis d'acquérir 2800 ha en plaine de Crau.

Pour parer au contexte de crise de la filière ovine, les éleveurs tentent de valoriser cette production sous signes de qualité (label rouge ou IGP) ou en vente directe mais restent cependant fortement dépendants des aides européennes, notamment des mesures agroenvironnementales. Dans un contexte économique difficile pour la filière ovine et porté par ce regain d'intérêt des politiques publiques pour l'élevage, le rôle de l'éleveur évolue peu à peu vers celui de gestionnaire d'un agroécosystème aux multiples objectifs : productif toujours, mais aussi environnemental et culturel. L'éleveur est de plus en plus considéré comme le garant de l'intégrité d'un écosystème assumé comme anthropisé et dont le fonctionnement est intimement lié aux pratiques d'élevage. Le retour du loup dans les Alpes tend à bouleverser cette dynamique. De pilote de l'écosystème, l'éleveur devient un intrus dans un écosystème considéré comme "naturel» par la société. Ce hiatus est accentué par le fait que les attentes diffèrent d'un territoire à l'autre. En plaine, le rôle environnemental de l'élevage reste fort, notamment via la prévention des risques d'incendie. À l'alpage en revanche, le berger, obligé d'adapter son système à la présence du loup (parcage nocturne, chiens de protection) lâche peu à peu prise sur ses missions environnementales avec un abandon du pâturage dans 
les espaces les plus inaccessibles et un surpâturage aux abords des zones de parcage et voit sa relation avec les touristes se dégrader notamment du fait de la multiplication des morsures de randonneurs par les chiens de protection.

\section{3 / Synthèse et leviers d'action}

Prairies humides et parcours méditerranéens constituent des systèmes multifonctionnels où élevage et milieux seminaturels sont intimement liés. L'élevage utilise les ressources naturelles du milieu et gère les habitats et le paysage. Ces systèmes utilisent assez peu d'intrants, induisent peu d'emplois directs mais sont responsables du maintien du tissu rural et du développement d'activités touristiques (figure 3 et figure 5). Les marchés sont réduits mais les produits de qualité et caractérisés par une identité territoriale peuvent bénéficier d'une bonne valorisation. Dans ces deux cas, l'élevage per se porte des enjeux culturels et via l'entretien de milieux ouverts est garant de milieux patrimoniaux. Ces deux systèmes sont néanmoins fragiles, les surfaces de prairies de marais ont été drastiquement réduites du fait de l'assèchement pour la mise en place de cultures céréalières, ou de l'abandon des parcelles les plus contraignantes à exploiter. Aujourd'hui encore, la menace de tels changements d'usage de terre est forte en Europe et le maintien des prairies à forte valeur conservative n'est pas assuré. L'élevage ovin transhumant méditerranéen est également menacé, par une pression foncière grandissante sur les espaces de Crau d'une part, et par une conjoncture croisant retour du loup et crise de la filière d'autre part ${ }^{2}$.

La gestion des milieux à haute valeur patrimoniale est parfois réalisée par les organismes chargés de la gestion des espaces protégés qui se substituent aux agriculteurs en gérant leurs propres troupeaux. Parfois la gestion est réalisée par une contractualisation avec un agriculteur qui s'engage, en contrepartie d'un faible loyer à mettre en place des pratiques extensives. Ces solutions adoptées ponctuellement, ne peuvent constituer une solution durable et généralisable à la gestion de prairies semi-naturelles fournissant des services écosystémiques majeurs comme la séquestration de carbone, la gestion des crues et la prévention des incendies. Le maintien de formes d'élevage économiquement viables dans des systèmes à exploitation contrainte représente ainsi un véritable enjeu, qui ne peut être réalisé que par une politique agroenvironnementale adaptée qui reconnaisse et rémunère les services rendus à travers des aides financières publiques.

Ces territoires à forts enjeux naturels s'appuient sur une gestion par des systèmes d'élevage extensifs, souvent en situation de difficulté économique. Ils sont de ce fait soumis à la double menace de l'intensification et de l'abandon. L'enjeu est alors d'améliorer les performances économiques de ces systèmes sans dégrader la qualité environnementale des milieux. L'exemple de l'Irlande (Sullivan et al 2010) montre en quoi l'intensification de l'usage des prairies basé sur un recours accru à la fertilisation (même en conservant un système fortement herbager) peut nuire à la biodiversité prairiale à de larges échelles. Il est alors nécessaire de proposer des leviers d'action alternatifs à une " simple » hausse de la fertilisation dans une logique d'agroécologie (Dumont et al 2013). Au niveau de l'exploitation, cela peut passer par une meilleure valorisation des ressources fourragères en repensant la gestion du troupeau par rapport à la pousse de l'herbe, en ajustant les périodes de vêlages/d'agnelages ou en jouant sur les complémentarités entre espèces herbivores dans des systèmes mixtes. Cela peut également passer par la recherche d'une plus grande autonomie et un objectif de minimisation des charges de l'exploitation. Aux niveaux supra-exploitation, l'enjeu est double : d'une part soutenir les dynamiques écologiques aux larges échelles, d'autre part viser une meilleure valorisation du produit en intégrant à sa valeur les dimensions environnementales et culturelles du produit. Sur le premier aspect, de nombreux travaux ont montré l'importance des échelles supra-exploitation dans les dynamiques écologiques de ces milieux et des dynamiques collectives commencent à se mettre en place pour la gestion écologique des paysages. C'est par exemple le cas de la MAE " mosaic management » aux Pays-Bas dans le cadre de laquelle les éleveurs raisonnent collectivement l'utilisation des prairies afin de favoriser la reproduction de la barge à queue noire (Shekermann et al 2008, Oosterveld et al 2011). Audelà de ces mesures collectives visant à limiter l'impact environnemental, les éleveurs peuvent également s'organiser collectivement pour mieux intégrer à la valeur de leur produit les dimensions culturelles et environnementales. Cela peut se faire par le recours aux indications géographiques (Benoit et Méda 2017, ce numéro) mais aussi en jouant plus directement sur la dimension environnementale des systèmes d'élevage considérés. Dans les basses vallées angevines (Billaudeau et Thareau 2010) par exemple, un collectif d'éleveurs tente depuis plusieurs années de valoriser leur production en associant explicitement l'élevage à son effet positif sur la biodiversité via une marque collective « l'éleveur et l'oiseau ».

\section{Références}

Badan O., Brun J.P., Congès G., 1995. Les bergeries romaines de la Crau d'Arles : Les origines de la transhumance en Provence. Gallia, 52, 263-310.

Benhammou F., 2007. Crier au loup pour avoir la peau de l'ours - Une géopolitique locale de l'environnement à travers la gestion et la conservation des grands prédateurs en France. Thèse de l'école doctorale ABIES, 665p.

Benoit M., Méda B., 2017. Enjeux et atouts des productions animales sous signe officiel de qualité pour répondre aux attentes sociétales. In : Numéro spécial, L'élevage en Europe : une diversité de services et d'impacts. Dumont B (Éd). INRA Prod. Anim., 30, 381-394.

Billaudeau V., Thareau B., 2010. L'éleveur et l'oiseau : rayonnement d'une démarche agroenvironnementale innovante. In : Économie sociale et solidaire, nouvelles trajectoires d'innovations. Boutiller S., Allemand S. (Éds). L'Harmattan, Paris, France, 155-187.

Bonis A., 2004. Recherches en prairies naturelles de marais. In : Valeur fourragère et écologique des prairies de marais. Compte-rendu de la journée d'échanges entre agriculteurs et scientifiques. Forum des Marais Atlantiques. Rochefort, France, 2-17.

Bonis A., Bouzillé J.B. Dausse A., Dia A. Hénin O., Bouhnik-Le Coz M., 2008. Fertilisation et qualité de l'eau en prairies naturelles humides (marais de l'Ouest). Fourrages, 196, 485-489.

Boujot C., 2003. De terre et d'eau. Au rythme de la blanchie dans les marais du Cotentin. CRECET, Caen, France, 88p.

Bouzillé J.B., 1992. Structure et dynamique des paysages, des communautés et des populations

2 S'il est indéniable que le loup a un impact réel sur les troupeaux, il est cependant nécessaire de rappeler que cet impact s'inscrit dans un contexte de crise profonde de la filière ovine. La question est alors de savoir si le loup constitue une menace suffisante pour mettre à lui seul en péril les systèmes d'élevage ou s'il n'est pas plutôt un révélateur des limites des profondes transformations que la filière ovine a subit au cours des dernières décennies (Benhammou 2007). 
végétales des marais de l'Ouest. Thèse d'Etat. Université de Rennes 1, Rennes, France, 282p.

Bouzillé J.B., Kerneis E., Bonis A., Touzard B., 2001. Vegetation and ecological gradients in abandoned salt pans in western France. J. Veg. Sci., 12, 269-278.

Bremond P., Grelot F., Agenais A.L., 2013. Economic evaluation of flood damage to agriculture - review and analysis of existing methods. Nat. Hazards Earth Syst. Sci., 13, 2493-2512.

Buisson E., Dutoit T., 2006. Creation of the natural reserve of La Crau: Implications for the creation and management of protected areas. J. Environ. Manage., 80, 318-326.

Bullock A., Acreman M., 2003. The role of wetlands in the hydrological cycle. Hydrol. Earth Syst. Sc., 7, 358-389.

Debout G., Fillol N., Wetton J.B., 2003. Les oiseaux nicheurs des prairies humides. Parc naturel régional des marais du Cotentin et $\mathrm{du}$ Bessin, Les Veys, France, 48p.

Diquelou S., Fillol N., Juhel C., LemauvielLavenant S., 2016. Biodiversité et valeur agronomique dans les prairies humides des marais du Cotentin et du Bessin. Erica, 30, 51-58.

Dumont B., Fortun-Lamothe L., Jouven M., Thomas M., Tichit M., 2013. Prospects from agroecology and industrial ecology for animal production in the $21^{\text {st }}$ century. Animal, 7, 10281043

Dumont B., Ryschawy J., Duru M., Benoit M., Delaby L., Dourmad J.Y., Méda B., Vollet D., Sabatier R., 2017. Les bouquets de services, un concept clé pour raisonner l'avenir des territoires d'élevage. In : Numéro spécial, L'élevage en Europe : une diversité de services et d'impacts. Dumont B. (Éd). INRA Prod. Anim., 30, 407-422.

Duncan P., Hewison A.J.M., Houte S., Rosoux R., Tournebize T., Dubs F., Burel F., Bretagnolle V., 1999. Long-term changes in agricultural practices and wildfowling in an internationally important wetland, and their effects on the guild of wintering ducks. J. Appl. Ecol., 36, 11-23.

Durant D., Tichit M., Kerneis E., Fritz H., 2008. Management of agricultural wet grasslands for breeding waders: integrating ecological and livestock system perspectives - a review. Biodivers. Conserv., 17, 2275-2295.

Duru M., Donnars C., Ryschawy J., Therond O., Dumont B., 2017. La «grange »: un cadre conceptuel pour appréhender les bouquets de services rendus par l'élevage dans les territoires. In : Numéro spécial, L'élevage en Europe : une diversité de services et d'impacts. Dumont B. (Éd). INRA Prod. Anim., 30, 273-284.

Fillol N., Diquélou S., Lemauviel-Lavenant S., 2015. Valeur fourragère des prairies de marais Approche comparée des sites du programme WOW. Rapport WP 4.2: WOW Project, 33p.

Gardiner T., 2009. Conserving Culm grassland Corby. British Naturalists' Association, 14p.

Gaujour E., Amiaud B., Mignolet C., Plantureux S., 2012. Factors and processes affecting plan biodiversity in permanent grasslands. A review. Agron. Sustain. Dev., 32, 133-160.

Halada L., Evans D., Romao C., Petersen J.E., 2011. Which habitats of European importance depend on agricultural practices? Biodivers Conserv., 20, 2365-2378.

Henle K., Alard D., Clitherow J., Cobb P., Firbank L., Kull T., McCracken D., Moritz R., Niemela J., Rebane M., Wascher D., Watt A. Young J., 2008. Identifying and managing the conflicts between agriculture and biodiversity conservation in Europe - A review. Agricult. Ecosys. Environ., 124, 60-71.

Huyghe C., De Vliegher A., Van Gils B. Peeters, A., 2014. Grasslands and herbivore production in Europe and effects of common policies. Editions Quae, Versailles, France, 287p.

Maltby E., Acreman M.C., 2011. Ecosystem services of wetlands: pathfinder for a new paradigm. Hydrolog. Sci. J., 56, 1341-1359.

Maltby E., Acreman M., Blackwell M.S.A. Everard M., Morris J., 2013. The challenges and implications of linking wetland science to policy in agricultural landscapes - experience from the UK National Ecosystem Assessment. Ecol. Eng., 56, 121-133.

Menichino N.M., Fenner N., Pullin A.S., Jones P.S., Guest J., Jones L., 2016. Contrasting response to mowing in two abandoned rich fen plant communities. Ecol. Eng., 86, 210-222.

Moreira F., Rego F.C., Ferreira P.G., 2001 Temporal (1958-1995) pattern of change in cultural landscape of northwestern Portugal implications for fire occurrence. Landscape Ecol., 16, 557-567.

Oosterveld E.B., Nijland F., Musters C.J.M., de Snoo G.R., 2011. Effectiveness of spatial mosaic management for grassland breeding shorebirds. J. Ornithol., 152, 161-170.

Opdekamp W., Beauchard O., Backx H., Franken F., Cox T.J.S., van Diggelen R., Meire P., 2012. Effects of mowing cessation and hydrology on plant trait distribution in natural fen meadows. Acta Oecol., 39, 117-127.

Ostermann O.P., 1998. The need for management of nature conservation sites designated under Natura 2000. J. Appl. Ecol., 35, 968-973.

Patthey P., Signorell N., Rotelli L., Arlettaz R. 2012. Vegetation structural and compositional heterogeneity as a key feature in Alpine black grouse microhabitat selection: conservation management implications. Eur. J. Wildl. Res. $58,59-70$.

Pelen J.N., 1985. Le pays d'Arles : sentiments d'appartenance et représentation de l'identité. Terrain 5, 37-45

Petri M., Batello. C., Villani R., Nachtergaele F., 2010. Carbon status and carbon sequestration potential in the world's grasslands. In Grassland carbon sequestration: management, policy and economics. Abberton M., Conant R., Batello C. (Eds). FAO, Rome, Italie, 19-31.

Poschlod P., Bakker J.P., Kahmen S., 2005. Changing land use and its impact on biodiversity. Basic Appl. Ecol., 6, 93-98.

Reine R., Barrantes O., Chocarro C., Juarez A., Broca A., Maestro M., Ferrer C., 2014. Pyrenean meadows in Natura 2000 network: grass production and plant biodiversity conservation. Span. J. Agricult. Res., 12, 61-77.

Sabatier R., Doyen L., Tichit M., 2010. Modelling trade-offs between livestock grazing and wader conservation in a grassland agroecosystem. Ecol. Model., 221, 1292-1300.

Schekkerman H., Teunissen W., Oosterveld E., 2008. The effect of "mosaic management" on the demography of black-tailed godwit Limosa limosa on farmland. J. Appl. Ecol., 45, $1067-$ 1075.

SQW, 2013. Impact of flooding on key business sectors in Devon and Somerset 2012-2013. Final report. Londres, Grande Bretagne, 40p.

Sullivan C.A., Skeffington M.S., Gormally M.J., Finn J.A., 2010. The ecological status of grasslands on lowland farmlands in western Ireland and implications for grassland classification and nature value assessment. Biol. Conserv. $143,1529-1539$

Tillier S., 2011. Gérer durablement la foret méditerranéenne : exemple du parc naturel régional des Alpilles. Thèse. Université du Maine, Le Mans, France, 396p

Vincent M., 2011. Les alpages à l'épreuve des loups. Éditions Quae, Paris, France, 352p.

\section{Résumé}

La conservation de nombreux écosystèmes patrimoniaux est conditionnée par le maintien d'une activité d'élevage extensive. Les prairies humides et les systèmes transhumants de Méditerranée constituent deux exemples contrastés de territoires à forts enjeux naturels où excès d'eau dans le premier cas et sècheresses annuelles dans l'autre conditionnent à la fois la végétation et les conditions d'élevage. Les marais portent des services environnementaux majeurs comme l'accueil de la biodiversité, notamment de l'avifaune ou la régulation des crues. Ce sont des territoires complexes où de nombreux acteurs interagissent autour de la gestion des niveaux d'eau. Parmi eux, les éleveurs jouent un rôle clé en gérant des prairies contraignantes à exploiter. Les prairies de marais sont fragilisées par un double mouvement d'intensification et de déprise agricole. L'élevage craven, exemple de système ovin transhumant de la plaine de Crau en zone méditerranéenne, permet de lutter contre la fermeture des milieux et joue un rôle de protection contre certains risques naturels comme les incendies dans les collines en Provence ou contre les risques d'avalanche dans les Alpes. Dans la plaine de la Crau, les éleveurs sont au cœur des enjeux de biodiversité dans la conservation du « Coussoul », dernière steppe européenne. Crise de la filière ovine, retour du loup et pression foncière grandissante sur les espaces de Crau menacent ce système d'élevage. 
Le maintien de l'élevage représente un véritable enjeu pour la conservation des espaces à forte valeur patrimoniale. Il dépend de la viabilité économique de formes d'élevage soumises à de fortes contraintes naturelles et nécessite donc une politique agroenvironnementale adaptée. La dynamique des territoires, à travers une valorisation de produits de qualité, apparaît également comme un levier pour préserver les activités d'élevage et conserver les habitats naturels.

\begin{abstract}
Multiple services delivered by semi-natural lands depend on breeding activities

Semi-natural grasslands present opportunities for delivering multiple services but their conservation is tightly dependent on livestock farming. Wetlands and steppes represent two contrasting examples of semi-natural lands for which water in excess or deficit drives both natural habitats and livestock farming. Wetlands deliver environmental services such as support of biodiversity, particularly birds and flood protection. They constitute complex territories as many stakeholders interact to manage water levels. Among them, livestock farmers play a key role by managing those flooded grasslands. Wet grasslands are still endangered by both conversion to improved grasslands or crops and abandonment due to the difficulties associated with exploiting constrained lands. In south east France, transhumant sheep breeding allows to maintain open vegetation that decreases the risk of both fires in the Alpilles hills and avalanche in the Alp mountains. In La Crau, maintaining Sheep grazing appears to be the only way to ensure the durability of the "Coussoul" habitat, the last steppe area in Europe. The crisis of sheep farming, return of wolves to the area and industrial development in La Crau all constitute a significant threat to this breeding system. The maintenance of breeding activities constitutes a challenge for the conservation of areas which have a high heritage value. Farming sectors face natural constraints which make management of the system more difficult and affect the economic viability, resulting in a need for an agroenvironmental policy. An increased market value of quality products, which are linked to production region, appears to be a lever to preserve livestock farming while conserving natural habitats.
\end{abstract}

LEMAUVIEL-LAVENANT S., SABATIER R., 2017. Quand l'élevage est garant de la conservation de milieux patrimoniaux. In : Numéro spécial, L'élevage en Europe : une diversité de services et d'impacts. Dumont B. (Éd). INRA Prod. Anim., 30, 351-362. 